\title{
Fluorescence cascades evoked by resonant interatomic Coulombic decay of inner-valence excited neon clusters
}

\author{
Andreas Hans ${ }^{\mathrm{a}}$, Ltaief Ben Ltaief ${ }^{\mathrm{a}}$, Marko Förstel ${ }^{\mathrm{b}}$, Philipp Schmidt ${ }^{\mathrm{a}}$, Christian Ozga ${ }^{\mathrm{a}}$, Philipp Reiß ${ }^{\mathrm{a}}$, \\ Xaver Holzapfel $^{\mathrm{a}}$, Catmarna Küstner-Wetekam ${ }^{\mathrm{a}}$, Florian Wiegandt ${ }^{\mathrm{c}}$, Florian Trinter ${ }^{\mathrm{c}}$, Uwe Hergenhahn ${ }^{\mathrm{b}, \mathrm{d}}$, \\ Till Jahnke ${ }^{\mathrm{c}}$, Reinhard Dörner ${ }^{\mathrm{c}}$, Arno Ehresmann ${ }^{\mathrm{a}}$, Philipp V. Demekhin ${ }^{\mathrm{a}}$, André Knie ${ }^{\mathrm{a}, *}$ \\ ${ }^{a}$ Institut für Physik und CINSaT, Universität Kassel, Heinrich-Plett-Str. 40, 34132 Kassel, Germany \\ ${ }^{b}$ Max-Planck-Institut für Plasmaphysik, Wendelsteinstr. 1, 17491 Greifswald, Germany \\ ${ }^{c}$ Institut für Kernphysik, J. W. Goethe Universität, Max-von-Laue-Str. 1, 60438 Frankfurt, Germany \\ ${ }^{d}$ Leibniz Institute of Surface Modification, Permoserstr. 15, 04318 Leipzig, Germany
}

\begin{abstract}
Resonant interatomic Coulombic decay (RICD) in inner-valence excited neon clusters is observed by a combination of vacuumultraviolet (VUV) and UV/visible fluorescence spectroscopy. These ultrafast interatomic electronic processes efficiently quench radiation emission from inner-valence excited clusters. After RICD took place, outer-valence excited clusters relax further by emission of fluorescence. The direct correspondence of the structures observed in the VUV and UV/visible fluorescence signals implies that the final states of the spectator RICD decay by a cascade of radiative decays: First, by the Rydberg-to-Rydberg transitions in the UV/visible spectral range, and then, by the Rydberg-to-valence transition in the VUV range. Our study demonstrates a possibility of detecting interatomic electronic processes by UV/visible fluorescence spectroscopy.
\end{abstract}

Keywords: Electronic excitation and relaxation, Radiationless transitions, Interatomic Coulombic decay, VUV and UV/visible fluorescence, Quenching

PACS: 33.35.+r, 33.50.-j, 36.40.Vz

\section{Introduction}

When weakly-bound systems, such as van-der-Waals or hydrogen-bounded clusters, interact with exciting-radiation, an efficient energy transfer between two neighboring species in a cluster may occur by an ultrafast interatomic electronic process. Since its discovery [1], interatomic and intermolecular Coulombic decay (ICD) and related processes have been subjects of comprehensive experimental and theoretical investigations [2, 3, 4, 5]. An important consequence of ICD is that the energy transferred to a neighbor is used to eject typically a low kinetic energy electron (the ICD electron). These electrons are proven to be genotoxic and may induce irreparable damage in living tissues [6]. It has also been shown experimentally $[7,8,9,10]$ and theoretically $[11,12,13]$, that an ICD process can be triggered by a resonant excitation of nobel gas dimers and clusters, a process termed RICD.

In all those experimental studies, ICD was probed in ultrahigh vacuum by analyzing the charged particles via electron and/or ion spectroscopy. Such charged particles, however, may not be used to prove ICD processes in dense media, like, e.g., in large clusters or biological matter, due to their very short mean free path. The orders of magnitude larger mean free path of photons opens a door to investigate interatomic processes inside dense media. Recently [14], we have demonstrated that

\footnotetext{
${ }^{*}$ Corresponding Author: André Knie, Universität Kassel;

Email address: knie@physik. uni-kassel.de (André Knie)
}

interatomic processes can also be studied by means of undispersed fluorescence spectroscopy. We found that RICD following the inner-valence excitation of neon clusters opens a particular fluorescence relaxation pathway. Resonant features observed in the time-resolved VUV fluorescence signal from clusters were found to be in one-to-one correspondence with those in the low-energy electron yield, which was measured simultaneously, providing thereby an unambiguous proof of ICD.

In the present work, by employing a similar technique we demonstrate that ICD can also be studied by detecting photons emitted in the UV/visible fluorescence range. In order to validate this detection technique, we combined it here with the detection of VUV photons, which has already been proven to be a probe for ICD [14]. However, as will become evident below, $\mathrm{UV} /$ visible fluorescence spectroscopy can be a separate tool to study interatomic processes, as suggested in the study of radiative relaxation of inner-shell excited argon clusters [15]. Simultaneous detection of the VUV and UV/visible fluorescence, realized here, allowed us to uncover a detailed relaxation route of the inner-valence excited clusters. The paper is organized as follows. The presently studied process and details of the experiment are described in Sec. 2. The experimental results are analyzed and discussed in Sec. 3. We conclude with a brief summary. 


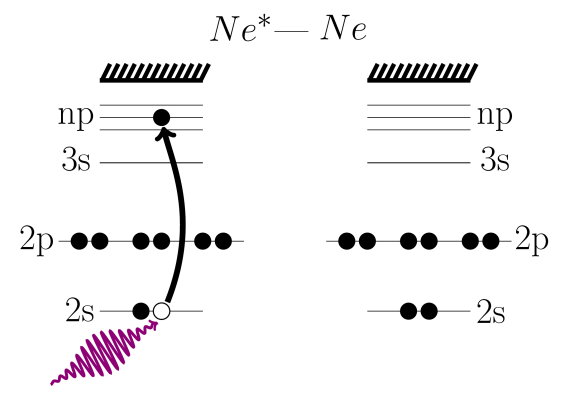

(a): Resonant excitation

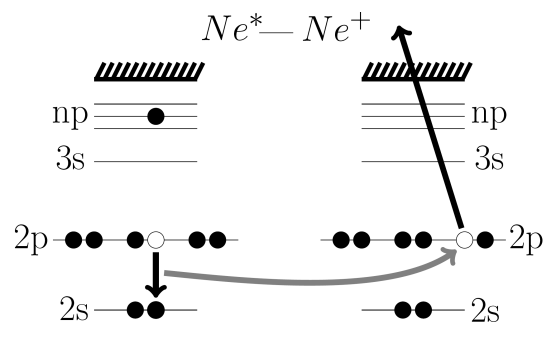

(b): Spectator RICD

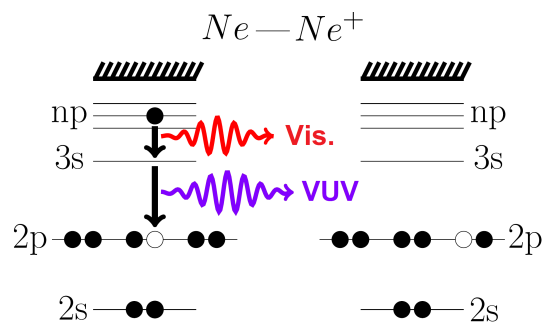

(c): Fluorescence cascade

Figure 1: The presently studied relaxation mechanism of inner-valence excited neon clusters, exemplified by dimers. Panel (a): the inner-valence 2s-electron of one $\mathrm{Ne}$ atom is resonantly promoted to an $n p$-Rydberg state by the absorption of an exciting-photon. Panel (b): the thus created $2 \mathrm{~s}-\mathrm{hole}$ is filled by an outer-valence $2 \mathrm{p}$-electron from the same excited atom, and the released energy is transferred to ionize a $2 \mathrm{p}$-electron of the neighboring Ne atom resulting in an ejection of a low kinetic energy ICD electron. Panel (c): after RICD, the excited Ne atom relaxes by a cascade of radiative decays, which consists of a $n p \rightarrow 3 s$ transition emitting a $\mathrm{UV} /$ visible photon and a subsequent $3 s \rightarrow 2 p$ transition releasing a VUV photon.

\section{Experiment}

The present experiment was carried out with the prototypical neon clusters. The process under consideration (schematically illustrated in Fig. 1) and the used experimental setup are very similar to those from our previous study of neon clusters [14]. At first (Fig. 1a), the resonant photon excites an innervalence electron of a $\mathrm{Ne}$ atom into the $n p$-Rydberg state. In an isolated atom, this excitation is followed by ultrafast autoionization, which usually dominates over radiative decay and thus suppresses any emission of fluorescence. Only for very high principle quantum numbers $n$, radiative decay can compete with autoionization [16]. Because of the presence of neighbors in the cluster, the inner-valence excited state can alternatively relax by the ICD process, in which the excess energy is transferred to a neighboring atom to release a slow ICD electron from it (Fig. 1b). As demonstrated theoretically [12] and verified experimentally [8] for $\mathrm{Ne}$ dimers, the efficiency of the spectator RICD for the $4 p$-excitation becomes comparable to, and for the $5 p$-excitation even dominant over, the autoionization. This efficiency grows with cluster size $[17,18]$.

After the spectator RICD took place, the originally excited atom still possesses a part of the excess energy, which is deposited in the Rydberg $n p$-electron and can only be released by the emission of a photon. Because the radiative decay rate grows significantly with the energy of the emitted photon, i.e., $\Gamma_{r} \sim \omega_{f l}^{3}$, one can expect that the Rydberg-to-valence $n p \rightarrow 2 p$ transition will dominate over the Rydberg-to-Rydberg $n p \rightarrow 3 s$ one (note that the former transition is forbidden only in systems with central symmetry). One thus would expect predominant fluorescence emission in the VUV range [14]. Here, we demonstrate that the Rydberg $n p$-electron relaxes by the fluorescence cascade (Fig. 1c). In spite of the above argumentation, the still excited $\mathrm{Ne}$ atom releases first a UV/visible fluorescence photon by the Rydberg-to-Rydberg transitions, and subsequently a VUV photon by the Rydberg-to-valence transition.

Experiments were performed at the synchrotron radiation facility SOLEIL (Paris) at the PLEIADES beamline in its multibunch operation mode. A slit-width of $300 \mu \mathrm{m}$ of the plane grating monochromator (PGM) was chosen to obtain a band- width of the exciting photons of about $12 \mathrm{meV}$ at $44 \mathrm{eV}$. The neon cluster jet is produced by supersonic expansion through a $30 \mu \mathrm{m}$ diameter nozzle separating the high pressure gas inlet from the vacuum in the expansion chamber and cooled by a liquid helium flow cryostat. For these measurements, the nozzle was cooled down to $75 \mathrm{~K}$, which, following Hagena's formulae [19], leads to mean cluster sizes of about $\langle N\rangle \sim 30$. After passing through a $1.5 \mathrm{~mm}$ diameter skimmer (BEAM DYNAMICS, INC.), the jet is transferred into the interaction chamber, where it is crossed with the linearly polarized photon beam of the monochromatized synchrotron radiation.

The UV/visible and VUV photons released in the second and third steps of the cascade (Fig. 1c) were simultaneously detected by the well-established set-up for photon-induced fluorescence-spectroscopy [20] (PIFS). It has already been successfully utilized to investigate autoionization $[21,22,23,24$, 25] and Auger decay [26, 27, 28, 29, 30] in molecules.

Briefly, an open-face stack of three micro-channel plates (MCP) was used to detect energetic ions and VUV photons with wavelength below $120 \mathrm{~nm}$. The stack is mounted in a differentially pumped chamber, separated from the interaction chamber by an aperture. The front of the MCPs is always set to $-2600 \mathrm{~V}$. Applying additionally $-150 \mathrm{~V}$ to a mesh in front of the stack, both energetic ions produced by Coulomb explosion (with kinetic energies of some eV [3]) and VUV photons are simultaneously detected. The former contribution to the recorded signal is typically by a factor of 10 larger than the later. In order to differentiate this total signal, the photon yield was acquired separately by setting the mesh voltage to $+200 \mathrm{~V}$, which repels all ions. The yield of energetic ions can thus be obtained by subtracting the photon yield from the total signal, both normalized to the acquisition time, as the photon flux was constant in top-up operation of the synchrotron.

The UV/visible fluorescence was collected using a MCP detector with bialkali photocathode and fused silica window, which covers the wavelength range from 300 to $630 \mathrm{~nm}$. The exciting-photon energy was varied in steps of $20 \mathrm{meV}$ in the wide range of $46.8-48.7 \mathrm{eV}$ (in steps of $10 \mathrm{meV}$ in the narrow range of $46.9-47.7 \mathrm{eV}$ ) and calibrated against the 2 s-ionization 


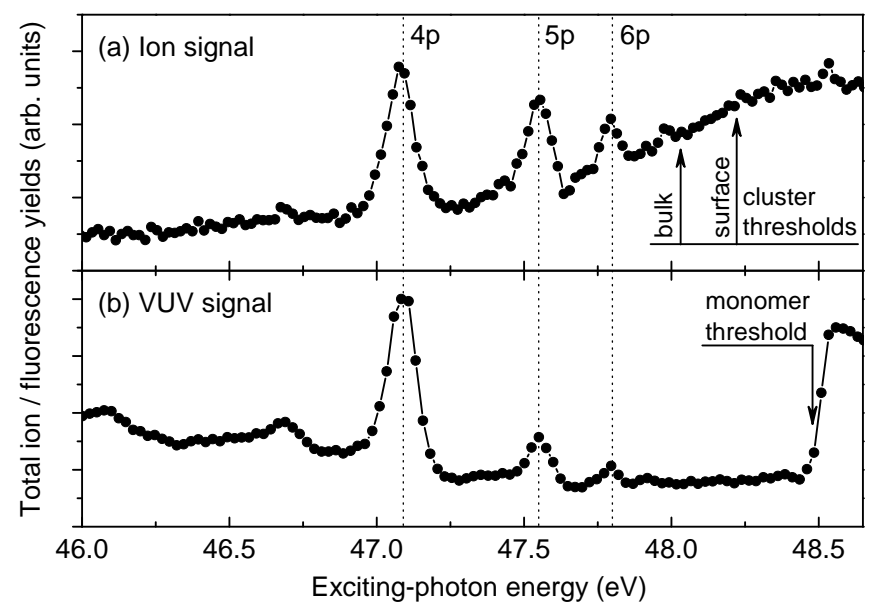

Figure 2: Overview of the total yield of energetic ions (panel a) and of the total VUV fluorescence yield (panel b) recorded in the vicinity of the inner-valence ionization of neon clusters. The $2 s \rightarrow n p$ resonances are assigned according to Ref. [31]. The ionization thresholds for neon monomers and clusters from Refs. [32] and [18] are indicated by the vertical arrows.

threshold of neon atoms at $48.48 \mathrm{eV}$. In a separate measurement performed at an exciting-photon energy of $47.09 \mathrm{eV}$, the VUV signal was dispersed by a 1-m-normal-incidence monochromator equipped with a gold-coated $1200 \mathrm{l} / \mathrm{mm}$ grating, achieving a resolution of about $\Delta \lambda \sim 2 \mathrm{~nm}$. The dispersed fluorescence was detected with a position sensitive open-face MCP detector as used in previous investigations [14].

\section{Results and Discussion}

The presently measured total yields of the ions and of the VUV fluorescence are compared in Fig. 2. In the shown exciting-photon energy range, the former exhibits three distinct resonant features and a broad ionization threshold [18] (see Fig. 2a). Those resonant structures were also observed in the spectrum of the low kinetic energy electrons in Ref. [7] and in the threshold photoelectron spectrum in Ref. [31]. According to the calculations performed in Ref. [31], they are assigned to the $2 s \rightarrow n p(n=4,5,6)$ resonant excitations in neon clusters. The inner-valence excited neon clusters and dimers relax predominantly by the spectator RICD $[7,8,11]$. The ultrafast electronic decay enables the subsequent relaxation of the still excited cluster by the emission of fluorescence [14]. This fact is illustrated in Fig. 2b. One can see that the presently measured total VUV fluorescence yield exhibits a resonant increase of the signal across the $2 s \rightarrow n p$ resonances.

The present cluster jet contains a considerable admixture of Ne atoms. These uncondensed monomers provide a clear contribution to the VUV signal in Fig. 2b at the exciting-photon energies above the atomic 2 s-ionization threshold at $48.48 \mathrm{eV}$ [32]. The observed signal corresponds to the $2 s^{-1} \rightarrow 2 p^{-1}$ VUV line in $\mathrm{Ne}$ [33] at about $46.1 \mathrm{~nm}$. Within the experimental uncertainties, we do not observe significant changes in the fluorescence signal in between the last cluster resonance and the atomic ionization threshold. This is because the inner-valence ionization threshold of clusters opens already at about $48.04 \mathrm{eV}$

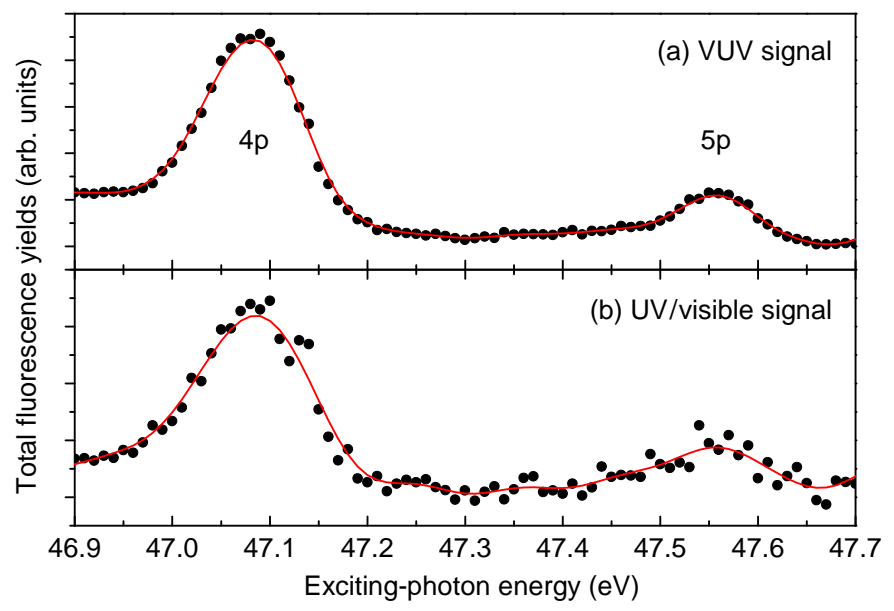

Figure 3: Total yields for the VUV fluorescence (panel a) and for the UV/visible fluorescence (panel b) recorded across the $2 s \rightarrow 4 p$ and $2 s \rightarrow 5 p$ resonant excitations in neon clusters. To guide the eye, the experimental data (circles) were additionally smoothed by FFT filter (lines). The resonances are assigned according to Ref. [31].

[18]. Being 2s-ionized, neon clusters decay by the normal ICD on the femtosecond timescale $[34,35]$ to the electronic ground state of the ion with almost $100 \%$ efficiency [36], quenching thereby an emission of the VUV fluorescence in cations.

Figure 3 compares the total VUV and UV/visible fluorescence yields of neon clusters recorded simultaneously with half the energy step size in a narrower energy interval. The $2 s \rightarrow 4 p$ and $2 s \rightarrow 5 p$ resonant excitations of Ne clusters emerging in the VUV signal (panel a) as a consequence of RICD are also clearly seen in the UV/visible fluorescence yield (panel b). The direct correspondence of the resonant structures observed in the VUV and UV/visible signals unequivocally suggests that the latter fluorescence emerges owing to RICD as well. This entails that the spectator RICD final states release their excess energy by a cascade of the radiative decays in the two different spectral ranges. From the energy consideration, a UV/visible photon can only be released by a Rydberg-to-Rydberg transition and solely in the first step of this cascade. The VUV photon, in turn, is emitted by a Rydberg-to-valence transition in the second relaxation step.

In order to trace the decay pathway during this radiative cascade, we have additionally dispersed the VUV fluorescence from neon clusters. This separate measurement was performed for the most intense $2 s \rightarrow 4 p$ resonance at the exciting-photon energy of $47.09 \mathrm{eV}$. The dispersed VUV fluorescence spectrum in the wavelength interval of $58-85 \mathrm{~nm}$ is depicted in Fig. 4. With the present wavelength resolution we observe only one fluorescence line at about $74 \mathrm{~nm}$. We do not observe any VUV signal with larger wavelengths (smaller photon energies), even in the wavelength range above $85 \mathrm{~nm}$ (not shown in the figure). We thus conclude that the observed fluorescence line corresponds to the relaxation of the lowermost outervalence excited state, i.e, to the $3 s \rightarrow 2 p$ transition in neon clusters. This cluster line is the analogue of the atomic lines $2 s^{2} 2 p^{5}\left({ }^{2} P_{1 / 2}\right) 3 s \rightarrow 2 s^{2} 2 p^{6}$ and $2 s^{2} 2 p^{5}\left({ }^{2} P_{3 / 2}\right) 3 s \rightarrow 2 s^{2} 2 p^{6}$ seen at about 73.59 and $74.37 \mathrm{~nm}$ [32], respectively, which sup- 


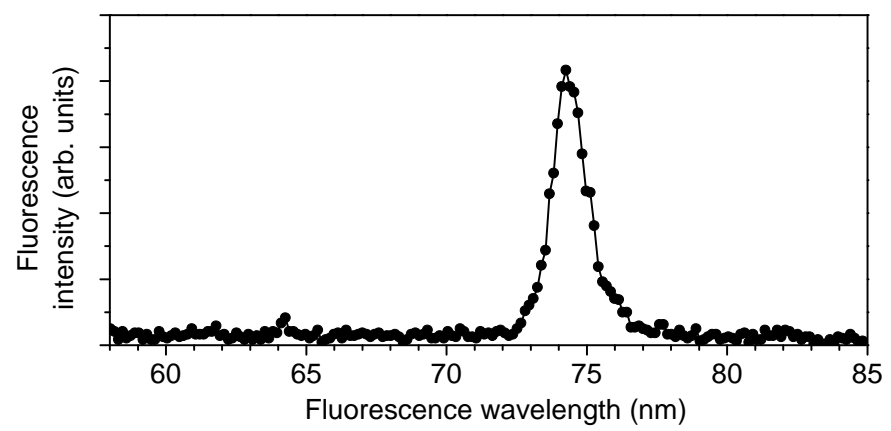

Figure 4: Dispersed VUV fluorescence spectrum of neon clusters recorded at the exciting-photon energy of $47.09 \mathrm{eV}$, which corresponds to the $2 s \rightarrow 4 p$ [31] electronic excitation in Fig. 3. The fluorescence signal in clusters observed around $74 \mathrm{~nm}$ is the analogue of the atomic transition line $2 s^{2} 2 p^{5}\left({ }^{2} P\right) 3 s \rightarrow$ $2 s^{2} 2 p^{6}$ in the $\mathrm{Ne}$ atom [32]

ports the presently suggested assignment.

Importantly, we do not observe any VUV signal with shorter wavelengths (larger photon energies). Such a fluorescence signal can be expected as the result of the direct relaxation of the spectator RICD final state implied in our previous work [14], i.e., by the radiative decay of the outer-valence exited cluster in its ground electronic state via the $4 p \rightarrow 2 p$ transition. According to atomic data tables [32], one would expect this VUV line around $61 \mathrm{~nm}$. The absence of the signal in the wavelength range between 58 and $72 \mathrm{~nm}$ (see Fig. 4) can only be explained by the validity of the dipole selection rules, which shows the atomic character of the excited states inside the cluster. As a consequence, the spectator RICD final state has only one possible relaxation pathway via the $n p \rightarrow 3 s$ Rydberg-to-Rydberg radiative decay in the $\mathrm{UV} /$ visible fluorescence range.

\section{Summary}

By combining VUV and UV/visible fluorescence spectroscopy and additionally dispersing the VUV fluorescence, we have experimentally observed a cascade of decays in the innervalence excited neon clusters. In the first step of this cascade, the spectator resonant interatomic Coulombic decay (RICD) efficiently quenches autoionization of the excited $\mathrm{Ne}$ atom and opens thereby a possibility for subsequent fluorescence relaxation pathways. In the second step, the spectator RICD final states emit UV/visible fluorescence by a transition to the lowest 3 s-Rydberg state. Finally, the VUV photon is released by the relaxation of this Rydberg electron into the ground state in the third step of the cascade of decays. We believe that UV/visible fluorescence spectroscopy may become a powerful tool for future investigations of ultrafast relaxation processes of excited systems in media and mechanisms of energy and charge transfer in general.

\section{Conflict of interest}

The authors declare that there is no conflict of interest.

\section{Acknowledgement}

This work was supported by the State of Hesse Initiative for the Development of Scientific and Economic Excellence (LOEWE) within the focus-project Electron Dynamics of Chiral Systems (ELCH) and by the Deutsche Forschungsgemeinschaft (Forschergruppe FOR 1789). We acknowledge the PLEIADES beamline team and the SOLEIL staff for excellent support during the experiments. We thank A.I. Kuleff, K. Gokhberg and L.S. Cederbaum for many valuable discussions.

\section{References}

[1] L.S. Cederbaum, J. Zobeley, F. Tarantelli, Phys. Rev. Lett. 79 (1997) 4778.

[2] V. Averbukh, Ph.V. Demekhin, P. Kolorenč, S. Scheit, S.D. Stoychev, A.I. Kuleff, Y.-C. Chiang, K. Gokhberg, S. Kopelke, N. Sisourat, L.S. Cederbaum, J. Electron Spectr. Relat. Phenom. 183 (2011) 36.

[3] U. Hergenhahn, J. Electron Spectrosc. Relat. Phenom. 184 (2011) 78.

[4] U. Hergenhahn, Intl. J. Radiat. Biology 88 (2012) 871.

[5] T. Jahnke, J. Phys. B 48 (2015) 082001.

[6] B. Boudaïffa, P. Cloutier, D. Hunting, M.A. Huels, L. Sanche, Science 287 (2000) 1658.

[7] S. Barth, S. Joshi, S. Marburger, V. Ulrich, A. Lindblad, G. Öhrwall, O. Björneholm, U. Hergenhahn, J. Chem. Phys. 122 (2005) 241102.

[8] T. Aoto, K. Ito, Y. Hikosaka, E. Shigemasa, F. Penent, P. Lablanquie, Phys. Rev. Lett. 97 (2006) 243401.

[9] F. Trinter, J.B. Williams, M. Weller, M. Waitz, M. Pitzer, J. Voigtsberger, C. Schober, G. Kastirke, C. Muüller, C. Goihl, P. Burzynski, F. Wiegandt, R. Wallauer, A. Kalinin, L.Ph.H. Schmidt, M.S. Schöffler, Y.-C. Chiang, K. Gokhberg, T. Jahnke, R. Dörner, Phys. Rev. Lett. 111 (2013) 233004.

[10] O'Keeffe, E. Ripani, P. Bolognesi, M. Coreno, M. Devetta, C. Callegari, M. Di Fraia, K.C. Prince, R. Richter, M. Alagia, A. Kivimäki, L. Avaldi, J. Phys. Chem. Lett. 4 (2013) 1797.

[11] K. Gokhberg, V. Averbukh, L.S. Cederbaum, J. Chem. Phys. 124 (2006) 144315.

[12] S. Kopelke, K. Gokhberg, L.S. Cederbaum, V. Averbukh, J. Chem. Phys. 130 (2009) 144103.

[13] G. Jabbari, S. Klaiman, Y.-C. Chiang, F. Trinter, T. Jahnke, K. Gokhberg, J. Chem. Phys. 140 (2014) 224305.

[14] A. Knie, A. Hans, M. Förstel, U. Hergenhahn, P. Schmidt, P. Reiß, C. Ozga, B. Kambs, F. Trinter, J. Voigtsberger, D. Metz, T. Jahnke, R. Dörner, A.I. Kuleff, L.S. Cederbaum, Ph.V. Demekhin, A. Ehresmann, New J. Phys. 16 (2014) 102002.

[15] I.L. Bradeanu, R. Flesch, M. Meyer, H.-W. Jochims, E. Rhl, Eur. Phys. J. D 36 (2005) 173.

[16] P. Lablanquie, F. Penent, R.I. Hall, J.H.D. Eland, P. Bolognesi, D. Cooper, G.C. King, L. Avaldi, R. Camilloni, S. Stranges, M. Coreno, K.C. Prince, A. Müehleisen, M. Žitnik, Phys. Rev. Lett. 84 (2000) 431.

[17] R. Santra, J. Zobeley, L.S. Cederbaum, Phys. Rev. B 64 (2001) 245104.

[18] G. Öhrwall, M. Tchaplyguine, M. Lundwall, R. Feifel, H. Bergersen, T. Rander, A. Lindblad, J. Schulz, S. Peredkov, S. Barth, S. Marburger, U. Hergenhahn, S. Svensson, O. Björneholm, Phys. Rev. Lett. 93 (2004).

[19] O.F. Hagena, Surf. Sci. 106 (1981) 101.

[20] H. Schmoranzer, H. Liebel, F. Vollweiler, R. Müller-Albrecht, A. Ehresmann, K.-H. Schartner, B. Zimmermann, Nucl. Inst. Meth. A 467-468 (2001) 1526.

[21] H. Liebel, S. Lauer, F. Vollweiler, R. Müller-Albrecht, A. Ehresmann, H. Schmoranzer, G. Mentzel, K.-H. Schartner, O. Wilhelmi, Phys. Lett. A 267 (2000) 357.

[22] H. Liebel, R. Müller-Albrecht, S. Lauer, F. Vollweiler, A. Ehresmann, H. Schmoranzer, J. Phys. B 34 (2001) 2581.

[23] H. Liebel, A. Ehresmann, H. Schmoranzer, Ph.V. Demekhin, B.M. Lagutin, V.L. Sukhorukov, J. Phys. B 35 (2002) 895.

[24] A. Ehresmann, L. Werner, S. Klumpp, H. Schmoranzer, Ph.V. Demekhin, B.M. Lagutin, V.L. Sukhorukov, S. Mickat, S. Kammer, B. Zimmermann, K.-H. Schartner, J. Phys. B 37 (2004) 4405.

[25] A. Ehresmann, Ph.V. Demekhin, W. Kielich, I. Haar, M.A. Schlüter, V.L. Sukhorukov, H. Schmoranzer, J. Phys. B 42 (2009) 165103. 
[26] A. Ehresmann, L. Werner, S. Klumpp, S. Lucht, H. Schmoranzer, S. Mickat, R. Schill, K.-H. Schartner, Ph.V. Demekhin, M.P. Lemeshko, V.L. Sukhorukov, J. Phys. B 39 (2006) 283.

[27] A. Ehresmann, W. Kielich, L. Werner, Ph.V. Demekhin, D.V. Omelyanenko, V.L. Sukhorukov, K.-H. Schartner, H. Schmoranzer, Euro. Phys. J. D 45 (2007) 235.

[28] Ph.V. Demekhin, I.D. Petrov, V.L. Sukhorukov, W. Kielich, P. Reiss, R. Hentges, I. Haar, H. Schmoranzer, A. Ehresmann, Phys. Rev. A 80 (2009) 063425; Erratum Phys. Rev. A 81 (2010) 069902E.

[29] Ph.V. Demekhin, I.D. Petrov, V.L. Sukhorukov, W. Kielich, A. Knie, H. Schmoranzer, A. Ehresmann, Phys. Rev. Lett. 104 (2010) 243001.

[30] A. Knie, M. Ilchen, Ph. Schmidt, Ph. Reiß, C. Ozga, B. Kambs, A. Hans, N. Müglich, S.A. Galitskiy, L. Glaser, P. Walter, J. Viefhaus, A. Ehresmann, Ph.V. Demekhin, Phys. Rev. A 90 (2014) 013416.

[31] R. Flesch, N. Kosugi, A. Knop-Gericke, E. Rühl, Z. Phys. Chem. 228 (2014) 387

[32] A. Kramida, Y. Ralchenko, J. Reader, NIST Atomic Spectra Database (National Institute of Standards and Technology, Gaithersburg, MD, 2012), http://physics.nist.gov/PhysRefData/ASD/index.html

[33] K.-H. Schartner, B. Möbus, G. Mentzel, A. Ehresmann, F. Vollweiler, H. Schmoranzer, Phys. Lett. A 169 (1992) 393.

[34] A.I. Kuleff, L.S. Cederbaum, Phys. Rev. Lett. 98 (2007) 083201.

[35] K. Schnorr, A. Senftleben, M. Kurka, A. Rudenko, L. Foucar, G. Schmid, A. Broska, T. Pfeifer, K. Meyer, D. Anielski, R. Boll, D. Rolles, M. Kübel, M.F. Kling, Y.H. Jiang, S. Mondal, T. Tachibana, K. Ueda, T. Marchenko, M. Simon, G. Brenner, R. Treusch, S. Scheit, V. Averbukh, J. Ullrich, C.D. Schröter, R. Moshammer, Phys. Rev. Lett. 111 (2013) 093402.

[36] M. Förstel, T. Arion, U. Hergenhahn, J. Electron Spectr. Relat. Phenom. 191 (2013) 16. 